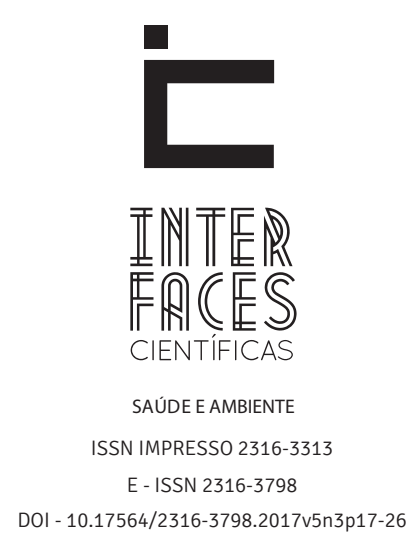

\title{
POLÍTICA PÚBLICA, DEFICIÊNCIA FÍSICA, CONCESSÃO DE ÓRTESES, PRÓTESES E MEIOS DE LOCOMOÇ̃̃O NO RIO GRANDE DO SUL: PERÍODO PRÉ/PÓS PLANO VIVER SEM LIMITES
}

\author{
PUBLIC POLICY, PHYSICAL DISABILITIES, ORTHOTICS, PROSTHESES AND MEANS OF LOCOMOTION CONCESSION IN RIO GRANDE DO \\ SUL: PERIOD PRE / POST PLAN LIVING WITHOUT LIMITS \\ POLITICAS PÚBLICAS, DISCAPACIDADES FÍSICAS, COCESIÓN ORTESIS, PRÓTESIS Y MEDIOS DE LOCOMOCIÓN EN RIO GRANDE DO SUL: \\ PERIODO PRE/POS PLANO VIVER SEM LIMITES
}

Christian Caldeira Santos ${ }^{1}$

Noberto Kuhn Junior
Valdir Pedde ${ }^{2}$

Jacinta Sidegum Renner

\section{RESUMO}

Objetivou-se caracterizar a Rede de Cuidados da Pessoa com Deficiência (RCPD), área Reabilitação Física, no Rio Grande do Sul e verificar se com a implantação do Plano Viver Sem Limites no estado, aumentou a concessão das Órteses, Próteses e Meios de Locomoção (OPML) às pessoas com deficiência física. Os dados foram obtidos no site IBGE (Censo 2010), no Portal da Saúde do Ministério da Saúde, na seção Relatórios da Gestão da Secretária de Atenção à Saúde, no site do Departamento de Informática do SUS (DATASUS), na plataforma Sistemas de Informações Ambulatoriais do SUS (SIA/SUS) e no Portal da Secretaria Estadual da Saúde, na seção Saúde da Pessoa com Deficiência. A RCPD no Rio Grande do Sul, na área da Reabilitação Física, cresceu e expandiu a partir do Plano Viver Sem Limites totalizando 11 Centros em Especializados em Reabilitação Física. A concessão de OPML no estado no período de 2008 a 2016, apresentou crescimento expressivo e estatisticamente significativo $(r=0,99 ; p$ $<0,0001)$. A concessão e os gastos com de OPML foi maior e estatisticamente significante $(p<0,001)$ no período Pós-Plano Viver Sem Limites (2012 a 2016). 
Esta política pública gerou resultados expressivos, facilitando o avançar na universalidade, equidade e integralidade aos mais de 800 mil usuários gaúchos com deficiência física.

\section{ABSTRACT}

Purpose of this study was to characterize the Network of Care of the Person with Disabilities (NCPD) in the Physical Rehabilitation area in Rio Grande do Sul and to verify if the implementation of the Living Without Limits Plan in the state increased the concession of Orthoses, Prostheses and Means of Locomotion (OPML) for people with physical disabilities. The data were obtained from the IBGE website (Census 2010), in the Health Portal of the Ministry of Health, in the section Reports on the Management of the Health Care Secretary, on the website of the Department of Information Technology of SUS (DATASUS) (SIA/SUS) and in the Portal of the State Health Secretariat, in the Health section of the Person with Disabilities. The NCPD in Rio Grande do Sul, in the area of Physical Rehabilitation, grew and expanded from the Living Without Limits Plan

\section{PALAVRAS-CHAVE}

Políticas Públicas, Deficiência, Órteses, Próteses, Equipamentos de Autoajuda.

totaling 11 Specialized Centers in Physical Rehabilitation. The granting of OPML in the state from 2008 to 2016 presented distinguished growth and statistically significant $(r=0.99 ; p<0.0001)$. The grant and expenditures with OPML were higher and statistically significant $(p<0.001)$ in the Post-Plan Living Without Limits period (2012 to 2016). This public policy generated expressive results, facilitating the advancement in universality, equity and integrality to the more than 800 thousand gaucho users with physical disabilities.

\section{KEYWORDS}

Public Policies. Disabilities. Orthoses, Prostheses. Self-Help Equipment.

\section{RESUMEN}

El objetivo fue caracterizar la Rede de Cuidados da Pessoa com Deficiência (RCPD), área de rehabilitación física en Rio Grande do Sul y verificar si, luego de la implementación del Plano Viver Sem Limites en el estado, hubo un aumento de la concesión de Ortesis, Prótesis y Medios de locomoción (OPML) para personas con discapacidad física. Los datos se obtuvieron del sitio de IBGE (Censo 2010), el portal de salud del Ministério da Saúde, la sección de Relatórios da Gestão da Secretária de Atenção à Saúde en la página web del SUS (DATASUS) en la plataforma de Sistemas de Informações Ambulatoriais do SUS (SIA/SUS) y el portal del no Portal da Secretaria Estadual da Saúde en la sección de salud de la persona con discapacidad. El RCPD en Rio Grande do Sul, en el área de rehabilitación física, ha crecido y expandido desde el Plano Viver Sem
Limites por un total de 11 centros de rehabilitación física especializada. La concesión de OPML en estado en el período de 2008 a 2016 mostró un crecimiento expresivo y estadísticamente significativo $(r=0,99 ; p<0,0001)$. La concesión y el gasto OPML fue mayor y estadísticamente significativa $(p<0,001)$ en el período posterior al Plano Viver Sem Limites (2012 a 2016). Esta política pública genera resultados significativos, facilitar el avance en la universalidad, equidad e integridad a los más de 800.000 usuarios gauchos con discapacidad.

\section{PALABRAS-CLAVE}

Políticas Públicas, Discapacidad, Ortesis, Prótesis, Equipo de Autoayuda. 


\section{INTRODUÇÃO}

No Brasil existem cerca de 45,6 milhões de pessoas que apresentam algum tipo de deficiência, o que em valor relativo totaliza $23,9 \%$ da sua população (IBGE, 2010a). Este conjunto de pessoas possui impedimentos crônicos de natureza física, mental, intelectual ou sensorial, os quais, em interação com outros obstáculos, podem subtrair e/ou neutralizar sua participação plena e efetiva na sociedade, em igualdade de condições com a população geral (BRASIL, 2015a).

Especificamente a deficiência físico/motora ocorre por afecção do sistema osteomuscular e/ou nervoso, e como consequências pode gerar déficits de funcionalidade e reciprocamente a necessidade de uso de Órteses, Próteses e Meios de Locomoção (OPML) (RIO GRANDE DO SUL, 2010).

Diante da debilidade funcional e das situações de desigualdades, opressão e discriminação sofridas por estas pessoas, entende-se que para o reconhecimento da cidadania deste segmento exige a adoção de políticas públicas capazes de aliar aspectos de justiça distributiva ao reconhecimento e respeito das mesmas como pares, de forma a possibilitarem suas participações na vida em sociedade em igualdade de condições com as demais pessoas (SENNA; LOBATO; ANDRADE, 2013). Segundo Costa e outros autores (2010), este enfoque intensifica a prática do conceito de inclusão social.

$\mathrm{Na}$ tendência de assegurar a inclusão social da pessoa com deficiência, o Estado brasileiro tencionou ações e esforços nas mais diferentes áreas governamentais, como da educação, da assistência social, do trabalho e emprego, de direitos humanos, de mobilidade urbana, da saúde, entre outras (BRASIL, 2011a). O montante financeiro investido para a implementação de Políticas de Atenção à Saúde da Pessoa com Deficiência até 2015 ultrapassou os R\$ 155 milhões (BRASIL, 2010; 2011b; 2012b; 2013; 2014; 2015b).

Como resultado destas ações e esforços, em 17 de novembro de 2011 (Decreto $n^{\circ} 7612 / 2011$ ), foi criado o Plano Nacional dos Direitos da Pessoa com Deficiência - Viver Sem Limites. Este Plano apresenta finalidade de promover, por meio da integração e articulação de políticas, programas e ações, o exercício pleno e equitativo dos direitos das pessoas com deficiência (BRASIL, 2011a).

Esta ação chancelou o compromisso do Brasil com as prerrogativas da Convenção sobre os Direitos das Pessoas com Deficiência, Organização das Nações Unidas (ONU), e foi ratificada pelo nosso Governo como emenda constitucional. O Plano comunga ações de todos estados da federação e previu um investimento de R\$ 7,6 bilhões até 2014, em ações em quatro grandes eixos: educação, inclusão social, acessibilidade e atenção à saúde (SÚMULA DO PROGRAMA..., 2014).

Na seara de suas diretrizes, tratou da ampliação e qualificação da Rede de Atenção à Saúde da Pessoa com Deficiência (RASPD), em especial os serviços de habilitação e reabilitação (BRASIL, 2011a). Assim, em 2012, foi instituída a Rede de Cuidados à Pessoa com Deficiência (RCPD) por meio da criação, amplificação e articulação de pontos de atenção à saúde, no âmbito do Sistema Único de Saúde (SUS) (BRASIL, 2012a). A Rede aporta um conjunto de táticas e serviços de assistência às necessidades particulares de pessoas com deficiência auditiva, física, visual, intelectual, múltiplas deficiências e ostomizadas, seja na promoção à saúde, identificação precoce de deficiências, prevenção dos agravos, tratamento e reabilitação (SÚMULA DO PROGRAMA..., 2014).

Estima-se que até 2015, mais de 10,5 milhões de pessoas foram beneficiadas pela RCPD (BRASIL, 2014; 2015b; 2016a) e o investimento financeiro, nos três primeiros anos, ultrapassou R\$ 1,1 bilhão (BRASIL, 2013; 2014; 2015b; 2016a). Mediante a este aporte financeiro foram criados em todo país Centros Especializados em Reabilitação (CER) e qualificação dos serviços já existentes, criação de oficinas ortopédicas e ampliação da oferta de OPML vinculados aos serviços de reabilitação física do SUS (SÚMULA DO PROGRAMA..., 2014).

Para Galvão, Barroso e Grutt (2013) a concessão de OPML é um direito de todo brasileiro e sem 
dúvida um fator indispensável para a promoção de qualidade de vida dos seus usuários, além de maximizar a independência do indivíduo nas atividades de vida diárias, práticas, educacionais, de lazer e do trabalho.

Atualmente no estado do Rio Grande do Sul, a RCPD é composta por 11 CER e três Oficinas Ortopédicas relacionados ao serviço de Reabilitação Física. Acredita-se que estas unidades estejam se capacitando para atenderem as 818 mil pessoas que possuem deficiência do tipo físico/motora no estado (IBGE, 2010b). Nesta perspectiva questionase após a implantação do Plano Viver Sem Limites: como foram configurados os CER especializados em Reabilitação Física no estado do Rio Grande do Sul? Houve aumento na oferta de OPML à população gaúcha com deficiência física?

Portanto, este estudo objetiva caracterizar a RCPD, junto a área da Reabilitação Física no estado Sul Rio Grandense e verificar se a implantação do Plano Viver Sem Limites favoreceu a concessão de OPML às pessoas com deficiência física.

\section{MÉTODO}

Este estudo se caracteriza por ser uma pesquisa exploratória, classificada como levantamento documental e bibliográfico, que trata de prospecção de documentos de órgãos públicos e bases de dados, leitura, análise e interpretação de periódicos e artigos científicos.

Os dados populacionais referentes à população geral com deficiência e a gaúcha com deficiência física/motora foram extraídos do site do Instituto Brasileiro de Geografia e Estatística (IBGE), Censo 2010. Os dados numéricos dos investimentos federais da Rede de Cuidados à Pessoa com Deficiência e do Plano Viver Sem Limites foram obtidos do Portal da Saúde do Ministério da Saúde, na seção Relatórios da Gestão da Secretária de Atenção à Saúde de 2010 a 2015.

Os dados sobre a concessão e gastos federais com OPML, especificamente as ortopédicas e os auxiliares de locomoção, referente ao período de 2008 a 2016 foram obtidos a partir do site do Departamento de Informática do SUS (DATASUS), na plataforma Sistemas de Informações Ambulatoriais do SUS (SAI/SUS). E os dados em relação ao Plano Estadual da Rede de Cuidados à Pessoa com Deficiência do Rio Grande do Sul foram extraídos do Portal da Secretaria Estadual da Saúde do Rio Grande do Sul, na seção Saúde da Pessoa com Deficiência.

Todos os dados foram tabulados e apresentados em tabelas por meio da análise descritiva. Na análise estatística, verificou-se a normalidades dos dados pelo Teste de Lilliefors, onde os mesmos tendenciaram à normalidade, e por esta natureza paramétrica, foi utilizado o Teste t de Student e o cálculo do coeficiente de correlação de Pearson. O programa BioEstat 5.3 foi usado para análise dos dados com nível de significância de $5 \%$.

\section{RESULTADO E DISCUSSÃO}

O Governo do Estado do Rio Grande do Sul, por meio da Resolução $n^{\circ}$ 283/13, aprovou em 8 de julho de 2013 o Plano Estadual da RCPD. Esta Resolução trouxe objetivos, diretrizes, metas e foi caracterizada por ser um instrumento estratégico e norteador para execução, monitoramento e avaliação das ações da saúde da pessoa com deficiência no SUS no estado, em consonância com o Plano Estadual de Saúde, que imbricou aos eixos norteadores (Educação, Saúde, Inclusão Social e Acessibilidade) do Plano Nacional Viver Sem Limites (RIO GRANDE DO SUL, 2013b).

Na gênese do Plano Estadual da RCPD, no primeiro semestre de 2013, houve participação das 19 Coordenadorias Regionais de Saúde (CRS) (divididas em sete Macrorregiões estaduais: Metropolitana, Missioneira, Norte, Sul, Centro-Oeste, Vales e Serra), as quais representaram as 30 Regiões de Saúde. Cada Região de Saúde do estado possui uma Comissão Intergestores Regional responsável pelo planejamento e pactuação regional. A elaboração dos Planos de Ação Macrorregionais ocorreu entre as Regiões de 
Saúde que se articularam, integraram e trabalharam coletivamente. Esta ação configurou a transformação da RASPD para a RCPD no estado do Rio Grande do Sul (RIO GRANDE DO SUL, 2013b).

Neste momento ocorreu o tensionamento de ações intra e intersetoriais dos gestores municipais e estaduais, que seguiram as recomendações federais do Ministério da Saúde para a efetuação da implantação da RCPD no Rio Grande do Sul. Este diálogo foi indispensável para o fortalecimento da Rede ao longo dos anos, pois, nos diálogos se encaminham os desejos e anseios do movimento social para a implantação das ações niveladoras de oportunidades e facilitar a inclusão social das pessoas com algum tipo de deficiência. O Censo 2010 apresentou, por amostragem, mais de $800 \mathrm{mil}$ pessoas com deficiência motora no estado do Rio Grande do Sul, dos quais 533 mil apresentaram alguma dificuldade motora, 240 mil grande dificuldade motora e quase 45 mil não conseguiam executar nenhuma atividade (IBGE, 2010b).

Para Silva e outros autores (2012), na cúria das políticas públicas de saúde que facilitam a inclusão social das pessoas com deficiência, deve contemplar a questão social, sem o desleixo da característica do cuidado. Portanto, quando o SUS concede OPML aos seus usuários, espera-se que esta ação gere possibilidades das pessoas com deficiência de serem (re)incluídas no meio educacional, laboral e recreacional. Nesta tendência, a RCPD do Rio Grande do Sul, na área de Reabilitação Física necessitou ser ampliada, passando de nove CER em 2013 para onze em 2016.

Infere-se que a construção destas unidades superou a meta da RCPC no estado, que entre 2012 e 2015 estipulou habilitar dez CER e implantar um melhor fluxo de acesso das pessoas com deficiências múltiplas e/ou graves às OPML dispensadas. Evidencia-se que a primeira meta foi atingida (RIO GRANDE DO SUL, 2013a), entretanto, o fluxo de acesso (segunda meta) não está totalmente implementado no estado, pois a Macrorregião Centro-Oeste, que abrange a $4^{\mathrm{a}}$ e a $10^{\mathrm{a}} \mathrm{CRS}$, não dispõe do seu CER regional, gerando dificuldade de acesso da população local para o recebimento de OPML. Segundo Caro e outros autores (2014), esta situação fomenta dificuldades no processo de reabilitação do usuário, ocasionado pelo longo trajeto, pelo estresse físico e emocional, contribuindo na não aceitação e aderência do paciente ao uso das OPML.

Assim, o CER se apresenta como o núcleo da Rede, faz-se estratégico na qualificação, regulação e criação de padrões mínimos para os cuidados às pessoas com deficiência, inclusive, em termos de acolhimento de diferenças e de humanização dos cuidados. Adjacente a alguns CER vinculados à Reabilitação Física, está a Oficina Ortopédica, um ponto fundamental no processo de reabilitação, pois nas oficinas se produzem e se realizam a confecção, adaptação e manutenção de OPML, para posterior concessão aos usuários (CAMPOS; SOUZA; MENDES, 2014).

Sob o ponto de vista do Plano Viver Sem Limites, a partir de 2012 o Governo Federal realizou investimento acima dos R\$309 milhões em estruturação de unidades de atenção especializada em saúde no país (BRASIL, 2012b; 2013; 2014; 2015b) e como resultado ao final de 2016, obteve um aumento de cinco vezes o número de OPML concedidas no Rio Grande do Sul em relação ao ano de 2010.

Nesse período, o aporte financeiro acima dos R\$ 780 milhões em ação de atenção à saúde da população para procedimentos de média e alta complexidade no país, fez crescer as dispensações de OPML no estado do Rio Grande do Sul (BRASIL, 2015b; 2014; 2013; 2012b). Assim, infere-se que o aumento das verbas federais destinadas tanto para parte estrutural (construção e expansão dos CER no estado), quanto para os procedimentos de médias e altas complexidades repercutiram a um maior aporte de OPML à população gaúcha com deficiência física.

A concessão de OPML no estado, em número total, em relação aos anos de 2008 a 2016, demonstrou um crescimento expressivo e estatisticamente significativo a cada ano $(r=0,99 ; p<0,0001)$. Acredita-se que a partir do aumento do número de CER no estado, 
aumentou a prescrição e a concessão de OPML à população gaúcha com deficiência física. Ao comparar a concessão de OPML nos períodos pré-Plano Viver Sem Limites (2008 a 2011) e pós-Plano (2012 a 2016), visualiza-se o aumento relevante de concessões de OPML ao final de 2016, período pós-Plano em relação ao período anterior (Tabela 1 ).

Tabela 1 - Evolução e comparação na concessão de OPML no período pré e pós Plano Viver Sem Limites no estado do Rio Grande do Sul

\begin{tabular}{|l|l|l|l|}
\hline \multicolumn{2}{|l|}{$\begin{array}{l}\text { Pré-Plano Viver } \\
\text { Sem Limites }\end{array}$} & \multicolumn{2}{l|}{$\begin{array}{l}\text { Pós-Plano Viver } \\
\text { Sem Limites }\end{array}$} \\
\hline Anos & $\begin{array}{l}\text { Concessão } \\
\text { de OPML }\end{array}$ & Anos & $\begin{array}{l}\text { Concessão } \\
\text { de OPML }\end{array}$ \\
\hline 2008 & 4.474 & 2012 & 15.656 \\
\hline 2009 & 8.492 & 2013 & 17.667 \\
\hline 2010 & 10.549 & 2014 & 20.026 \\
\hline 2011 & 11.277 & 2015 & 22.937 \\
\hline & & 2016 & 23.845 \\
\hline & \multicolumn{3}{|l}{} \\
\hline Total & $34.792^{*}$ & & $100.131^{*}$ \\
\hline Total geral de OPML Concedidas 134.923 \\
* p < 001 \\
Fonte: Brasil (2016b).
\end{tabular}

Em relação à dotação financeira para o custeio das OPML (valor aprovado por ano) no estado do Rio Grande do Sul entre os anos 2008 a 2016, houve também um aumento estatisticamente significativo nos valores gastos a cada ano $(r=0,98 ; p<$ 0,0001 ). Ao comparar o montante financeiro gasto com as OPML nos períodos pré-Plano Viver Sem Limites (2008 a 2011) e pós-Plano (2012 a 2016), obtém-se diferença estatisticamente significativa entre os dois períodos, conforme demostrado na Tabela 2.
Tabela 2. Evolução e comparação das dotações financeiras de OPML no período pré e pós Plano Viver Sem Limites, valores expressos em reais, no estado do Rio Grande do Sul

\begin{tabular}{|c|c|c|c|}
\hline \multicolumn{2}{|c|}{$\begin{array}{l}\text { Pré-Plano Viver } \\
\text { Sem Limites }\end{array}$} & \multicolumn{2}{|c|}{$\begin{array}{l}\text { Pós-Plano Viver } \\
\text { Sem Limites }\end{array}$} \\
\hline Anos & $\begin{array}{l}\text { Dotação } \\
\text { em OPML }\end{array}$ & Anos & $\begin{array}{l}\text { Dotação } \\
\text { em OPML }\end{array}$ \\
\hline 2008 & $2.776 .582,35$ & 2012 & $9.542 .918,22$ \\
\hline 2009 & $5.717 .839,51$ & 2013 & $10.604 .256,92$ \\
\hline 2010 & $6.717 .768,49$ & 2014 & $12.046 .389,11$ \\
\hline \multirow[t]{2}{*}{2011} & $6.826 .489,88$ & 2015 & $13.956 .030,44$ \\
\hline & & 2016 & $14.402 .374,43^{*}$ \\
\hline Total & $22.038 .680,23^{*}$ & & $60.551 .969,12^{*}$ \\
\hline \multicolumn{4}{|c|}{ Total geral de Dotação em OPML 82.590.649,35 } \\
\hline
\end{tabular}

Para Galvão, Barroso e Grutt (2013) o fato do SUS, por meio da Portaria SAS/MS n ${ }^{0}$ 661, de 2 de dezembro de 2010, ter reconhecido e regulamentado a aptidão dos terapeutas ocupacionais e fisioterapeutas a prescreverem as OPML relacionadas ao ato não cirúrgico, favoreceu a ampliação de concessão destes equipamentos à população. Tais profissionais estão contidos no processo de reabilitação, favorecendo a prescrição de equipamentos, pois compreendem as necessidades do usuário, delineiam o seu perfil e selecionam o produto mais indicado a cada paciente.

$\mathrm{Na}$ tentativa de justificar o menor aporte de OPML à população no Rio Grande do Sul no período pré- Plano Viver Sem Fronteiras, Castro, Andrade e Stone (2015) afirmaram que o estado gaúcho apresentava uma menor dependência do serviço público de saúde, visto as melhores condições socioeconômicas de sua população. Em contraste a esta afirmação, cita-se que a justificativa mais coerente para esta questão, 
que aponta tanto para o número de OPML concedidas quanto para seus custos, foi dada pelo próprio Governo do Estado ao reconhecer a carência de acesso para os pacientes com deficiências múltiplas e graves, redução de mobilidade e/ou impossibilitados de comparecer às unidades ambulatoriais para aquisição dos dispositivos previstos na tabela SUS, resultando numa crescente demanda judicial para aquisição dos mesmos (RIO GRANDE DO SUL, 2013a).

Diante das situações favoráveis e desfavoráveis que permeiam qualquer implementação de políticas públicas, pontua-se que a política de reabilitação no âmbito do SUS a partir da RCPD, trouxe mudanças significativas na teoria e na prática para o ato do cuidado à saúde da pessoa com deficiência, pois vinculou estes cuidados aos princípios e diretrizes do SUS (universalidade, equidade e integralidade da atenção à saúde) referente às atividades de promoção, proteção e recuperação da saúde. Neste tocante, os CER foram estrategicamente posicionados em locais que não possuíam, ou estavam aquém da oferta de serviço de reabilitação.

Espera-se então que, por meio da RCPD, tanto no Rio Grande do Sul, como nos demais estados ocorra com vistas à superação das lacunas assistenciais e organizacionais, entre as esferas da Atenção Primária à Saúde, Atenção Especializada e Atenção Hospitalar e de Urgência e Emergência, no que tange à dispensação de cuidados integrais à saúde das pessoas com deficiência. Sua consolidação é dependente da capacidade de articulação entre estes pontos de atenção (PEREIRA; MACHADO, 2016). Neste momento esperase efetivar a Portaria PT GM/MS n 1060 que desde 5 de junho de 2002 já apontava o direito de a pessoa com deficiência ser assistida no âmbito do SUS, frente as necessidades fundamentais e específicas de saúde por meio de ações de promoção, prevenção e reabilitação, incluindo a aquisição de recursos ópticos e OPML.

Portanto, após cinco anos da criação do Plano Nacional dos Direitos da Pessoa com Deficiência - Viver Sem Limites, Mendes (2014) afirma que por ele a RCPD se encontra num dinamismo intenso e rápido de implantação nas diversas regiões brasileiras, por meio de pactuação e conversações no interior do SUS e com a sociedade civil. Este empreendimento público converge a um ganho histórico no que diz a respeito aos direitos sociais e, neles, de acesso qualificado das pessoas com deficiência à saúde.

\section{CONCLUSÃO}

Com advento do Plano Nacional dos Direitos da Pessoa com Deficiência - Viver Sem Limites e o surgimento da RCPD, a prática da assistência à pessoa com deficiência se fortaleceu e demonstra com o avançar dos anos uma tendência de crescimento e desenvolvimento favorável, porém ainda inacabada.

A RCPD no Rio Grande do Sul expandiu ano a ano na tentativa de oferecer maior e melhor assistência a saúde aos mais 800 mil gaúchos com deficiência física. Os onze CER implantados no estado contribuem para uma frequência maior de concessões de OPML no período Pós-Plano Viver Sem Limites em relação ao período Pré-Plano.

Assim, considera-se que a política pública promovida pelo Plano Viver Sem Limites gerou resultados expressivos, e fomenta o avançar da universalidade, equidade e integralidade aos usuários com deficiência física no âmbito do SUS.

\section{REFERÊNCIAS}

\section{BRASIL. Mistério da Saúde, Portaria MS/GM no}

$\mathbf{1 . 0 6 0}$ de 5 de junho de 2002 - Política Nacional de Saúde da Pessoa com Deficiência. MS: Brasília, 2002. Disponível em: <http://bvsms.saude.gov.br/bvs/ saudelegis/gm/2002/prt1060_05_06_2002.html>. Acesso em: 22 dez. 2016.

BRASIL. Casa Civil. Decreto no 7.612, de 17 de novembro de 2011. Institui o Plano Nacional dos Direitos da Pessoa com Deficiência - Plano Viver sem Limite. Brasília, 2011a. Disponível em: <http://www. planalto.gov.br/CCIVIL_03/_Ato2011-2014/2011/ Decreto/D7612.htm>. Acesso em: 22 set. 2015. 
BRASIL. Ministério da Saúde. Secretaria de Atenção à Saúde. Relatório de Gestão 2010. MS: Brasília. 2011b. Disponível em: <http://portalarquivos.saude. gov.br/images/pdf/2014/abril/01/relatorio-degestao-sas-2010.pdf>. Acesso em: 22 dez. 2016.

BRASIL. Ministério da Saúde. Portaria nº 793, de 24 de abril de 2012. Institui a Rede de Cuidados à Pessoa com Deficiência no âmbito do Sistema Único de Saúde. Brasília, 2012a. Disponível em: <http:// bvsms.saude.gov.br/bvs/saudelegis/gm/2012/ prt0793_24_04_2012.html>. Acesso em: 22 set. 2015.

BRASIL. Ministério da Saúde. Secretaria de Atenção à Saúde. Relatório de Gestão 2011. MS: Brasília. 2012b. Disponível em: <http://portalarquivos.saude. gov.br/images/pdf/2014/abril/01/relatorio-degestao-sas-2011.pdf>. Acesso em: 22 dez. 2016.

BRASIL. Ministério da Saúde. Secretaria de Atenção à Saúde. Relatório de Gestão 2012. MS: Brasília. 2013. Disponível em: <http://portalarquivos.saude. gov.br/images/pdf/2014/abril/01/relatorio-degestao-sas-2012.pdf>. Acesso em: 22 dez. 2016.

BRASIL. Ministério da Saúde. Secretaria de Atenção à Saúde. Relatório de Gestão 2013. MS: Brasília. 2014. Disponível em: <http://portalarquivos.saude. gov.br/images/pdf/2014/abril/01/relatorio-degestao-sas-2013.pdf>. Acesso em: 22 dez. 2016.

BRASIL. Casa Civil. Lei n 13.146, de 6 de julho de 2015. Institui a Lei Brasileira de Inclusão da Pessoa com Deficiência (Estatuto da Pessoa com Deficiência). Brasília, 2015a. Disponível em: <http:// www.planalto.gov.br/ccivil_03/_ato2015-2018/2015/ Lei/L13146.htm>. Acesso em: 22 set. 2015.

BRASIL. Ministério da Saúde. Secretaria de Atenção à Saúde. Relatório de Gestão 2014. MS: Brasília. 2015b. Disponível em: <http://portalarquivos.saude. gov.br/images/pdf/2015/maio/04/Relat--rio-de-Gest-o-da-SAS-2014-Final.pdf>. Acesso em: 22 dez. 2016.
BRASIL. Ministério da Saúde. Secretaria de Atenção à Saúde. Relatório de gestão 2015. MS: Brasília. 2016a. Disponível em: <http://portalarquivos.saude. gov.br/images/pdf/2016/marco/31/Relat--rio-deGest--o-da-SAS-2015-Final.pdf>. Acesso em: 22 dez. 2016.

\section{BRASIL. Ministério da Saúde - Sistema de \\ informações ambulatoriais do SUS (SIA/SUS).}

2016b. Disponível em: <http://tabnet.datasus.gov. br/cgi/tabcgi.exe?sia/cnv/qaRS.def>. Acesso em: 27 mar. 2017.

CAMPOS, M.F., SOUZA, L.A.P.; MENDES, V.L.F. A rede de cuidados do Sistema Único de Saúde à saúde das pessoas com deficiência. Interface - Comunic., Saude, Educ., Botucatu, v.19, n.52, p.207-10, 2015.

CARO, C.C. et al. A dispensação de órteses, próteses e meios auxiliares de locomoção (OPM) no Departamento Regional de Saúde da $3^{a}$ Região do Estado de São Paulo. Cad. Ter. Ocup. UFSCar, São Carlos, v.22, n.3, p.521-529, 2014.

COSTA, V.S.P. et al. Social representations of the wheelchair for people with spinal cord injury. Rev. Latino-Am. Enfermagem, Ribeirão Preto, v.18, n.4, p.755-762, 2010.

GALVÃO, C.R.C.; BARROSO, B.I.L.; GRUTT, D.C. A tecnologia assistiva e os cuidados específicos na concessão de cadeiras de rodas no Estado do Rio Grande do Norte. Cad. Ter. Ocup. UFSCar, São Carlos, v.21, n.1, p.11-18, 2013.

IBGE (Instituto Brasileiro de Geografia e Estatística). Censo Demográfico 2010: Características gerais da população, religião e pessoas com deficiência. Brasília: IBGE, 2010a. Disponível em: <http://www. ibge.gov.br/home/estatistica/populacao/censo2010/ caracteristicas_religiao_deficiencia/default_ caracteristicas_religiao_deficiencia.shtm>. Acesso em: 22 set. 2015. 
IBGE - Instituto Brasileiro de Geografia e Estatística. Censo Demográfico 2010: Rio Grande do Sul: Pessoas com Deficiência - Amostra. Brasília: IBGE, 2010b. Disponível em: <http://www.ibge.gov.br/ estadosat/temas.php?sigla=rs\&tema=censodem og2010_defic>. Acesso em: 27 dez 2016.

MENDES, V.L.F. Saúde Sem Limites: Implantação da Rede de Cuidados à Saúde da Pessoa com Deficiência. Divulg. Saúde Debate., Rio de Janeiro, n.52, p.146-152, 2014.

PEREIRA, J.S.; MACHADO, W.C.A. Referência e contrarreferência entre os serviços de reabilitação física da pessoa com deficiência: a (des)articulação na microrregião Centro-Sul Fluminense, Rio de Janeiro, Brasil. Physis, Rio de Janeiro, v.26, n.3, p.1033-1051, 2016.

RIO GRANDE DO SUL. Secretaria Estadual de Saúde. Departamento de Assistência Hospitalar e Ambulatorial. Manual Operativo para Dispensação de Órteses, Próteses e Meios Auxiliares de Locomoção do Estado do Rio Grande do Sul. 71p. Porto Alegre: SES, 2010. Disponível em: <http://www.saude.rs.gov. br/upload/1338328684_PCD\%20Manual\%20de\%20 OPMs.df>. Acesso em: 26 dez. 2016.
RIO GRANDE DO SUL. Secretaria Estadual de Saúde. Plano Estadual de Saúde 2012 - 2015. SES: Porto Alegre, 2013a. Disponível em: <http://www.saude. rs.gov.br/upload/1382374302_PES\%2020122015\%20FINAL.pdf>. Acesso em: 26 dez. 2016.

RIO GRANDE DO SUL. Secretaria Estadual de Saúde. Resolução n 283/13 - CIB/RS. SES: Porto Alegre. 2013b. Disponível em: <http://www.saude.rs.gov. br/upload/1375202666_Resolucao\%20e\%20Plano. pdf>. Acesso em: 22 dez. 2016.

SENNA, M.C.M.; LOBATO, L.V. C.; ANDRADE, L.D. Proteção Social à Pessoa com Deficiência no Brasil Pós-Constituinte. Ser Social, Brasília, v.15, n.32, p.11-33, 2013.

SILVA, R.A. et al. Políticas Públicas para Inclusão Social na Deficiência - Revisão Sistemática. Av. Enferm., Bogotá, v.30, n.2, p.13-24, 2012.

SÚMULA DO PROGRAMA “VIVER SEM LIMITES”: PLANO NACIONAL DOS DIREITOS DA PESSOA COM DEFICIÊNCIA. Card. Cedes, Campinas, v.34, n.93, p.263-266, 2014.
Recebido em: 8 de Novembro de 2016 Avaliado em: 11 de Dezembro de 2016 Aceito em: 14 de Dezembro de 2016

\footnotetext{
1 Docente do curso de Fisioterapia da Universidade Federal do Pampa - Unipampa; Discente do Programa de Pós-graduação em Diversidade Cultural e Inclusão Social, Universidade Feevale, Rio Grande do Sul, Brasil. Email: christiansantos@unipampa.edu.br

2 Docentes do Programa de Pós-graduação em Diversidade Cultural e Inclusão Social, Universidade Feevale, Rio Grande do Sul, Brasil. E-mail: valpe@feevale.br

3 Docentes do Programa de Pós-graduação em Diversidade Cultural e Inclusão Social, Universidade Feevale, Rio Grande do Sul, Brasil. E-mail: nkjunior@feevale.br

4 Docentes do Programa de Pós-graduação em Diversidade Cultural e Inclusão Social, Universidade Feevale, Rio Grande do Sul, Brasil. E-mail: jacinta@feevale.br
} 
